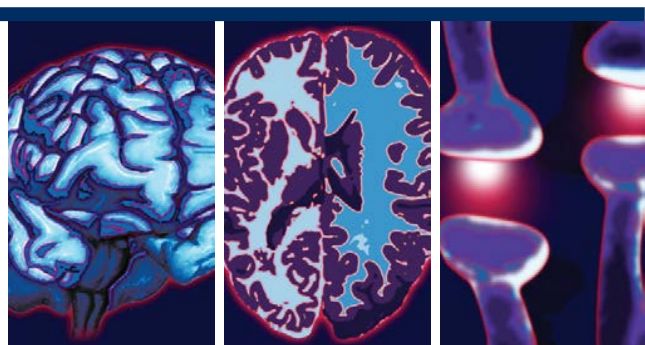

\title{
Effect of Memantine on Brain Metabolic Activity and Perfusion in Drug-naïve Moderate Alzheimer's Disease Patients
}

Tomo Iwamoto', Kumiko Utsumi ${ }^{2, \dagger}$, Seiju Kobayashi ${ }^{1,3}$, Shuichi Yasumura², Shigeki Hatakeyama' ${ }^{1}$, Ayako Hayashi ${ }^{3}$, Chiaki Kawanishi' ${ }^{1}$

\begin{abstract}
Objective: Memantine is a noncompetitive N-methyl-D-aspartate receptor (NMDAR) antagonist that improves or stabilizes cognitive impairment in moderate to severe Alzheimer's disease (AD). However, the effects of memantine on regional brain metabolic activity and perfusion are not fully known. To clarify these effects, we investigated the efficacy of memantine monotherapy using multimodal neuroimaging in drug-naïve patients with moderate AD.

Methods: This was a prospective open-labeled study of patients with drug-naïve moderate AD (Mini-Mental State Examination scores of 14-19) before and after 12 weeks of treatment with memantine, conducted between April 2015 and December 2016. Imaging was performed using 18F-fluorodeoxyglucose-positron emission tomography (18F-FDG-PET) and 99mTcethyl cysteinate dimer-single photon emission computed tomography (99mTc-ECD-SPECT), to assess brain metabolic activity and perfusion, respectively. The imaging data, registered to a probabilistic anatomical atlas, were evaluated by voxel-based analysis.
\end{abstract}

Results: A total of 20 patients were enrolled and 17 patients' datasets were analyzed. The average Mini-Mental State Examination was 16.6 (1.8) at baseline and 16.9 (4.1) posttreatment. The average Neuropsychiatric Inventory score was 2.8 (7.6) at baseline and 3.9 (9.2) post-treatment. Brain regions with increased metabolic activity following memantine treatment in previously drug-naïve $A D$ patients included a wide range of cerebral cortexes, particularly the right inferior parietal lobule, right supramarginal gyrus, right angular gyrus, and right paracentral lobule $(p<0.01$, paired t-test). Only small regions had increased brain perfusion ( $p<0.01$, paired t-test).

Conclusion: We believe this is the first study focusing on brain metabolic activity and perfusion in the same drug-naïve moderate AD patients before and after memantine treatment. There were inconsistencies between the regions with increased metabolic activity and perfusion after memantine treatment in drug-naïve $A D$ patients, suggesting that brain metabolism may increase without a concurrent increase in blood perfusion. This study may help elucidate the mechanism of action of memantine.

\section{Keywords}

Alzheimer's disease, memantine, 18F-FDG, PET, 99mTc-ECD, SPECT, MMSE, NPI

\footnotetext{
'Department of Neuropsychiatry, Sapporo Medical University, School of Medicine, Sapporo, Japan

${ }^{2}$ Department of Psychiatry, Sunagawa City Medical Center, Sunagawa, Japan

${ }^{3}$ Department of Psychiatry, Nakae Hospital, Sapporo, Japan

${ }^{\dagger}$ Author for correspondence: Kumiko Utsumi, MD, PhD, Department of Psychiatry, Sunagawa City Medical Center, 1 - 1, Nishi 4, Kita 3, Sunagawa, Japan, Tel: +81-125-54-2131, Fax: +81-125-54-0101; e-mail: 2017utsumi@gmail.com
} 


\section{Introduction}

Alzheimer's disease (AD) is the most common dementia, and the development of drug therapies for $\mathrm{AD}$ has advanced in recent years. Based on the pathophysiology hypothesis of $\mathrm{AD}$, two drug types, N-methyl-D-aspartate receptor (NMDAR) antagonists and cholinesterase inhibitors (ChEIs), have been used clinically in $\mathrm{AD}$ patients to date.

Memantine, the only noncompetitive NMDAR antagonist, has been used clinically, to improve or stabilize cognitive impairment in moderate to severe $\mathrm{AD}[1,2]$. However, the effects of memantine on regional brain metabolic activity and perfusion are not fully known.

Regional perfusion on single photon emission computed tomography (SPECT) is typically reduced in the parietal, temporal lobe, and posterior cingulate regions of $\mathrm{AD}$ patients [37]. 18F-fluorodeoxyglucose-positron emission tomography (18F-FDG-PET) measures regional cortical metabolic activity and has found that the metabolic rate in the parietal and temporal cortex is reduced early in the course of $\mathrm{AD}[8,9]$. Cortical metabolism declines as Alzheimer's disease progresses [10-13].

It has been reported that the degree of uptake on 18F-FDG-PET and 99mTc-ethyl cysteinate dimer-SPECT (99mTc-ECD-SPECT) in patients with $\mathrm{AD}$ and mild cognitive impairment shows significant correlations in the frontal, temporal, and parietal lobes [14].

Many previous studies have measured perfusion and metabolic activity in $\mathrm{AD}$ patients treated with ChEIs. ChEIs treatment increases cortical blood flow in the frontal lobe $[15,16]$, the right anterior cingulate, the dorsolateral prefrontal, and the temporoparietal areas bilaterally [17]. Another study demonstrated that ChEIs preserve cortical blood flow in right middle temporal gyrus [18] and the occipital precuneus [19]. Treatment with ChEIs has a positive effect on cerebral metabolism in the frontal region [20].

However, no previous study has measured changes in regional brain perfusion in patients with $\mathrm{AD}$ before and after memantine treatment. Additionally, studies about brain metabolic activity measured by $18 \mathrm{~F}-\mathrm{FDG}-\mathrm{PET}$ in $\mathrm{AD}$ patients treated with memantine are a few. Sultzer et al. reported that metabolic activity in the bilateral inferior temporal gyri and angular gyri and supramarginal gyri increased after 10 weeks of memantine treatment in patients with
$\mathrm{AD}$ on stable ChEI medication [21]. However, no previous study has simultaneously examined 18F-FDG-PET and 99mTc-ECD-SPECT on the same drug-naïve $\mathrm{AD}$ patients after memantine treatment alone.

It remains to be clarified how change in brain metabolic activity and perfusion occurs in previously drug-naïve $\mathrm{AD}$ patients after memantine treatment alone. Another question is whether the effects of memantine treatment assessed by brain functional imaging such as $99 \mathrm{mTc}$-ECD-SPECT and 18F-FDGPET are similar. To answer these questions, we investigated the efficacy of 12 weeks of memantine monotherapy using multimodal imaging (18F-FDG-PET and 99mTc- ECDSPECT) in drug-naïve patients with moderate $\mathrm{AD}$.

\section{Materials and Methods \\ n Study design}

This study was conducted between April 2015 and December 2016. Drug-naïve patients with moderate $\mathrm{AD}$ underwent imaging assessments with 18F-FDG-PET and 99mTc- ECDSPECT. Each patient then received open-label treatment with memantine for 12 weeks, and the clinical assessments and imaging assessments were repeated after the treatment.

\section{- Participants}

Twenty participants (6 men and 14 women) who had been diagnosed with Alzheimer's disease $(\mathrm{AD})$ were recruited from the outpatients of Sunagawa City Medical Center Hospital for Psychiatry, Sunagawa, Japan. The inclusion criteria were: 1) patients who met the clinical diagnosis of $\mathrm{AD}$ based on the criteria of both the Diagnostic and Statistical Manual of Mental Disorders 5th edition (DSM-5) and the National Institute on Aging-Alzheimer's Association (NIA-AA); and 2) patients with baseline MMSE scores of 14 to 19 .

Participants were excluded if they had the following: dementia due to other than Alzheimer's disease; evidence of other neurologic or psychiatric disorders; any medication with central nervous system activity; having serious health problems, and abnormal results of biochemical analysis that may affect cognition. All candidate patients were examined by experienced psychiatrists and received full clinical assessment, which included standard dementia screening with the Mini-Mental State 
Examination (MMSE), routine blood tests with complete blood count, biochemistry, thyroid function tests, vitamin levels, standard urine analysis. MMSE and Neuropsychiatric Inventory (NPI) assessments were made for each patient on the day they visited for SPECT scans. Informed written consent was obtained from all included participants and their relatives.

This study was carried out according to the Declaration of Helsinki. Each participant's privacy was protected, and the protocol was approved by the Ethics and Radiation Safety Committees of Sunagawa City Hospital, Sunagawa, Japan.

\section{- Memantine treatment}

On the day following 18F-FDG-PET imaging, patients started taking open-label memantine 5 mg once daily. The dosage was increased over 4 weeks, rising $5 \mathrm{mg}$ per week to a final dosage of $20 \mathrm{mg}$ once daily.

\section{- Clinical assessments}

Each participant's dementia symptoms were assessed using the MMSE and NPI. The MMSE was included as an overall measure of cognitive impairment. The NPI assesses behavioral and psychological disturbances occurring in patients with dementia. Both the severity and frequency of each symptom were measured, and this information was obtained from a caregiver familiar with the patient [22].

\section{- 18F-FDG-PET imaging}

PET imaging of cerebral metabolic activity in the resting state was performed using a Discovery PET/CT 600 scanner (GE Health care, Milwaukee, WI, USA). Each participant received an intravenous injection of 169.5-325.6 MBq 18F-FDG purchased from Nihon MediPhysics Co., Ltd. Participants rested quietly in a dimly-lit room during the 40-minute uptake phase. They were then positioned symmetrically dorsally in the scanner. For the acquisition of PET imaging, a 15-minute emission scan in list-mode was performed after the CT scan for attenuation correction. PET images were reconstructed with 3D ordered subset expectation maximization (VUE Point HD).

\section{n 99mTc- ECD-SPECT imaging}

Each participant received a 444.0-1085.0 $\mathrm{MBq}$ intravenous injection of $99 \mathrm{mTc}$-ECD as a commercially supplied kit (Neurolite injection Daiichi; Fujifilm RI Pharma, Japan) while lying down with their eyes closed in a quiet room. Nine minutes after the injection of $99 \mathrm{mTc}-\mathrm{ECD}$, brain SPECT was performed for 20 minutes using an E.CAM Signature Series scanner (Toshiba Medical Systems Corporation, Tokyo, Japan) equipped with a low energy high resolution collimator. Projection data were obtained in continuous mode, for 90 steps of $360^{\circ}$ at $4^{\circ}$ per step. The scanned data were prefiltered with a Butterworth filter (order 8 and a cut off at 0.11 cycles/ pixel). Brain images were reconstructed with filtered back projection. Attenuation correction was performed using Chang's method.

\section{- Imaging and data analyses}

PET and SPECT data were analyzed using PMOD 3.5 software (PMOD Technologies, LLC, Switzerland) and statistical parametric mapping 8 (SPM8; Wellcome Trust Centre for Neuroimaging) in Matlab 8 (MathWorks, Inc. Natick, MA, USA). Initially, all imaging data after the reconstruction was re-positioned, and then cortical metabolic activities were corrected by the standardized uptake value (SUV) of 18F-FDG using PMOD. PET images were normalized to Montreal Neurological Institute (MNI) atlas space using the PET template in SPM8 and smoothed using an $8 \mathrm{~mm}$ Gaussian filter. SPECT data were also normalized to MNI atlas space using the SPECT template in SPM8 smoothed with an $8 \mathrm{~mm}$ Gaussian filter. The global mean uptake in the entire brain was estimated by region-of-interest analysis referring to the Automated Anatomical Labeling (AAL) atlas, and then the SPECT brain perfusion pattern was evaluated by the whole brain uptake ratio (WBR). PET and SPECT images in the baseline condition (pre-memantine treatment) were compared with the post-treatment condition (after 12 weeks of memantine treatment) using the paired test procedure in SPM8, with $\mathrm{p}<0.01$ for PET and SPECT at the voxel level, respectively. In addition to the SPM analysis, volume of interest (VOI) analysis was performed utilizing VOIs defined in the PMOD AAL atlas. Each PET (SUV) and SPECT (WBR) value of each VOI was obtained from normalized images. SUV and WBR values between baseline and post treatment were compared using paired t-tests.

\section{- Statistical analysis}

Statistical analyses were performed with the $\mathrm{R}$ Statistical Software Package version 3.1.0 (R Core Team. Foundation for Statistical Computing, Vienna, Austria). Descriptive statistics and 
frequency distributions of baseline demographics and cognitive scores were summarized. Data from clinical assessments were analyzed using paired t-tests for comparison between baseline and after 12 weeks of memantine treatment. Statistical significance was set at $\mathrm{p}<0.01$.

\section{Results}

\section{- Patient Characteristics}

Of the 20 patients enrolled three were excluded from the analysis: One patient was not administrated with memantine at first visit, one lacked second scan data due to health reasons not related to memantine administration, and the third was revealed to have Lewy body dementia. The other 17 patients consisted of 6 men and 11 women, with an average age of 80.1 (6.2) years old. At baseline the mean (standard deviation [SD]) onset age was 78.3 (6.7) years old, mean education 9.5 (1.2) years, and the average body weight 55.3 (13.5) kg. The average MMSE was $16.6(1.8)$ at baseline and 16.9 (4.1) posttreatment. The average NPI score was 2.8 (7.6) at baseline and 3.9 (9.2) post-treatment. The patient characteristics are shown in Table 1.

\section{- Changes in brain metabolic activity}

Brain regions showing significant increases in brain metabolism are highlighted in Figure 1. Significant increases in metabolic activity were observed in wide range of whole cerebral cortical areas. VOIs showing significant increases in SUV were observed in the right inferior parietal lobule, right supramarginal gyrus, right angular gyrus, and right paracentral lobule (Table 2).

\section{- Changes in cerebral blood flow}

Brain regions with significant increases in cerebral blood flow are shown in Figure 2. Although a wide range of whole cerebral cortexes showed significant increases in brain metabolism (Figure 1), the brain regions in which significant increases in cerebral blood flow occurred were quite limited. No regions had VOIs with significant increases in WBR.

\section{Discussion}

This study shows, for the first time, a significant increase in brain metabolic activity in wide range of whole cerebral cortexes in drug-naïve $\mathrm{AD}$ patients after 12-weeks of memantine

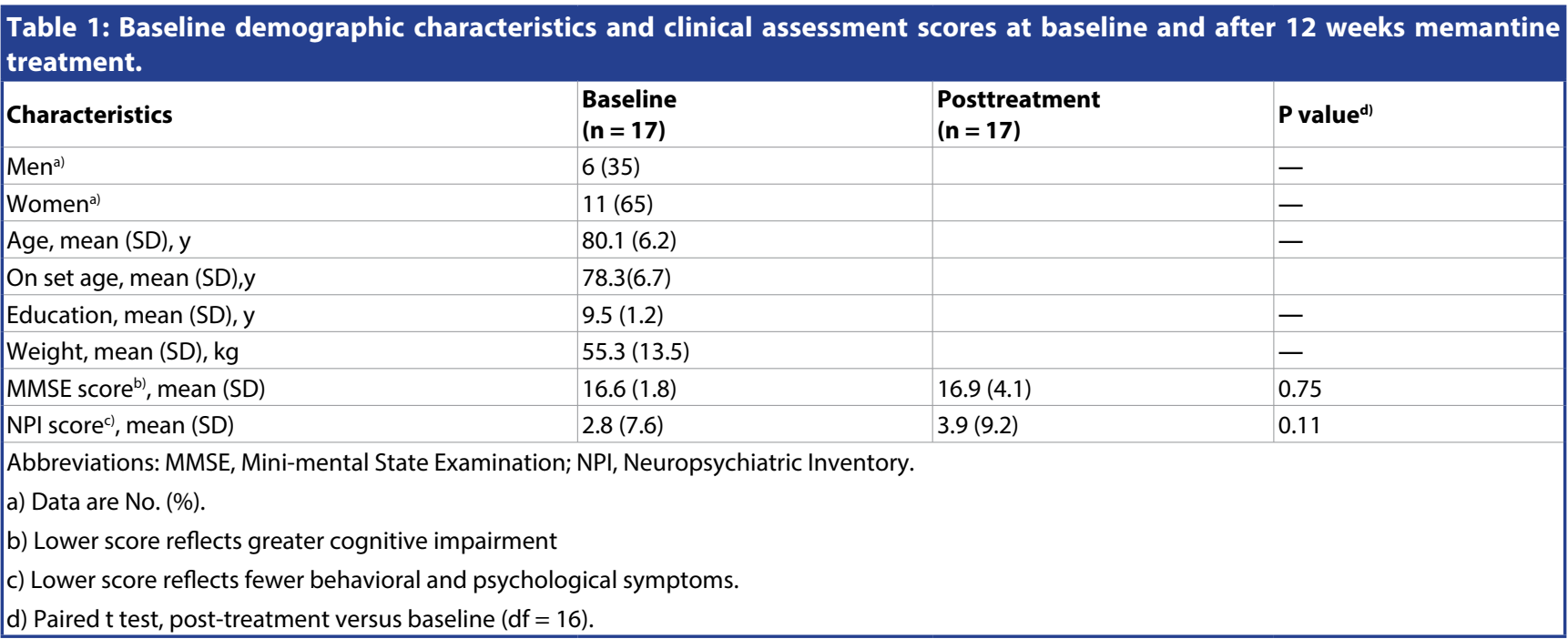

\begin{tabular}{|c|c|c|c|c|c|c|}
\hline \multirow{3}{*}{ VOI } & \multicolumn{3}{|c|}{ FDG-PET(SUV) } & \multicolumn{3}{|c|}{ ECD-SPECT(WBR) } \\
\hline & \multirow{2}{*}{ baseline } & \multirow{2}{*}{\begin{tabular}{|l|} 
post- \\
treatment
\end{tabular}} & \multirow{2}{*}{ P value ${ }^{a)}$} & \multirow{2}{*}{ baseline } & \multirow{2}{*}{\begin{tabular}{|l|} 
post- \\
treatment
\end{tabular}} & \multirow{2}{*}{ P value ${ }^{a)}$} \\
\hline & & & & & & \\
\hline Inf_Parietal_r & 6.07 & 7.04 & 0.009 & 0.98 & 0.98 & 0.952 \\
\hline Supra_Marginal_r & 5.99 & 6.84 & 0.009 & 1.05 & 1.06 & 0.445 \\
\hline Angular_r & 6.07 & 7.02 & 0.009 & 0.97 & 1.00 & 0.035 \\
\hline Paracentral_Lobule_r & 5.97 & 6.84 & 0.006 & 1.07 & 1.06 & 0.305 \\
\hline \multicolumn{7}{|c|}{$\begin{array}{l}\text { Abbreviations: SUV, standardized uptake value; WBR, whole brain ratio; Inf_Parietal_r, right inferior parietal lobule; Supra_Marginal_r, right } \\
\text { supramarginal gyrus; Angular_r, right angular gyrus; Paracentral_Lobule_r, right paracentral lobule; VOI, volume of interest. }\end{array}$} \\
\hline \multicolumn{7}{|c|}{ a) Paired t test, post-treatment versus baseline $(\mathrm{df}=16)$. } \\
\hline
\end{tabular}


Effect of Memantine on Brain Metabolic Activity and Perfusion in Drug-naïve Moderate Alzheimer's Disease Patients

(A)

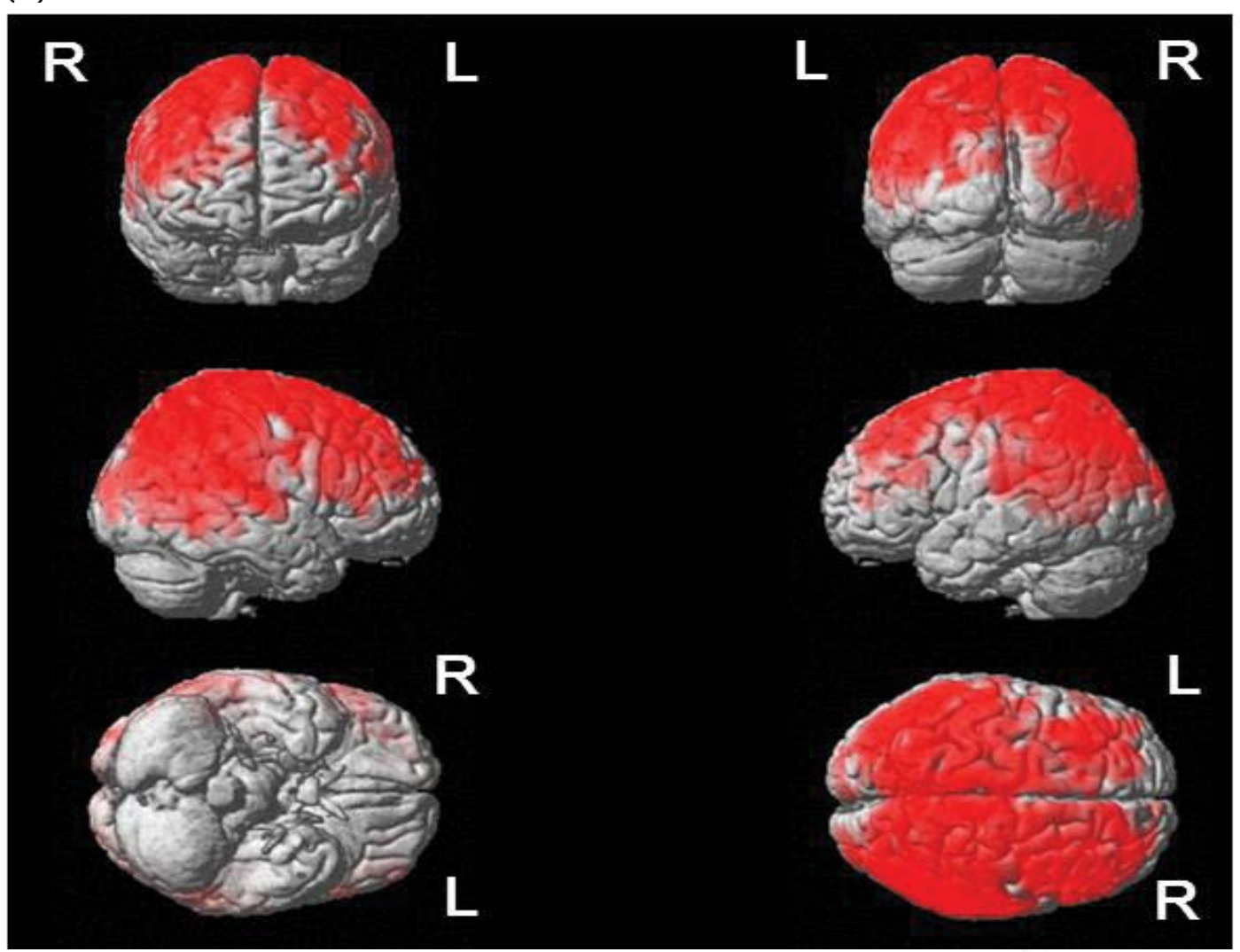

(B)
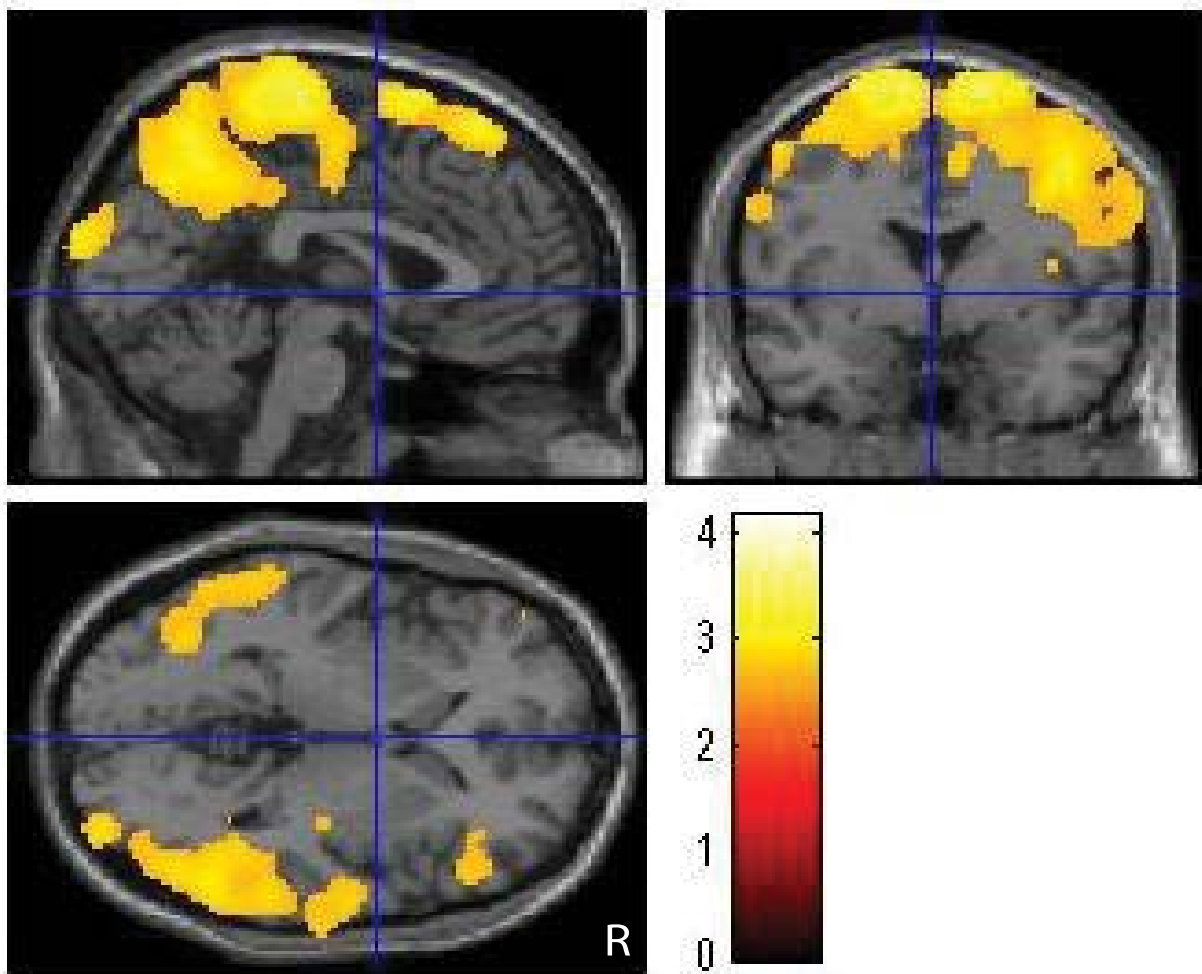

Figure 1: Statistical maps of higher metabolic activity after 12 weeks of memantine treatment compared with baseline. Regions with significantly higher metabolism ( $\mathrm{p}<0.01$; paired $t$-test) superimposed on a standard 3-dimensional anatomic template (3D-render) (A) and coregistered MRI slices (B). 
Research Kumiko Utsumi

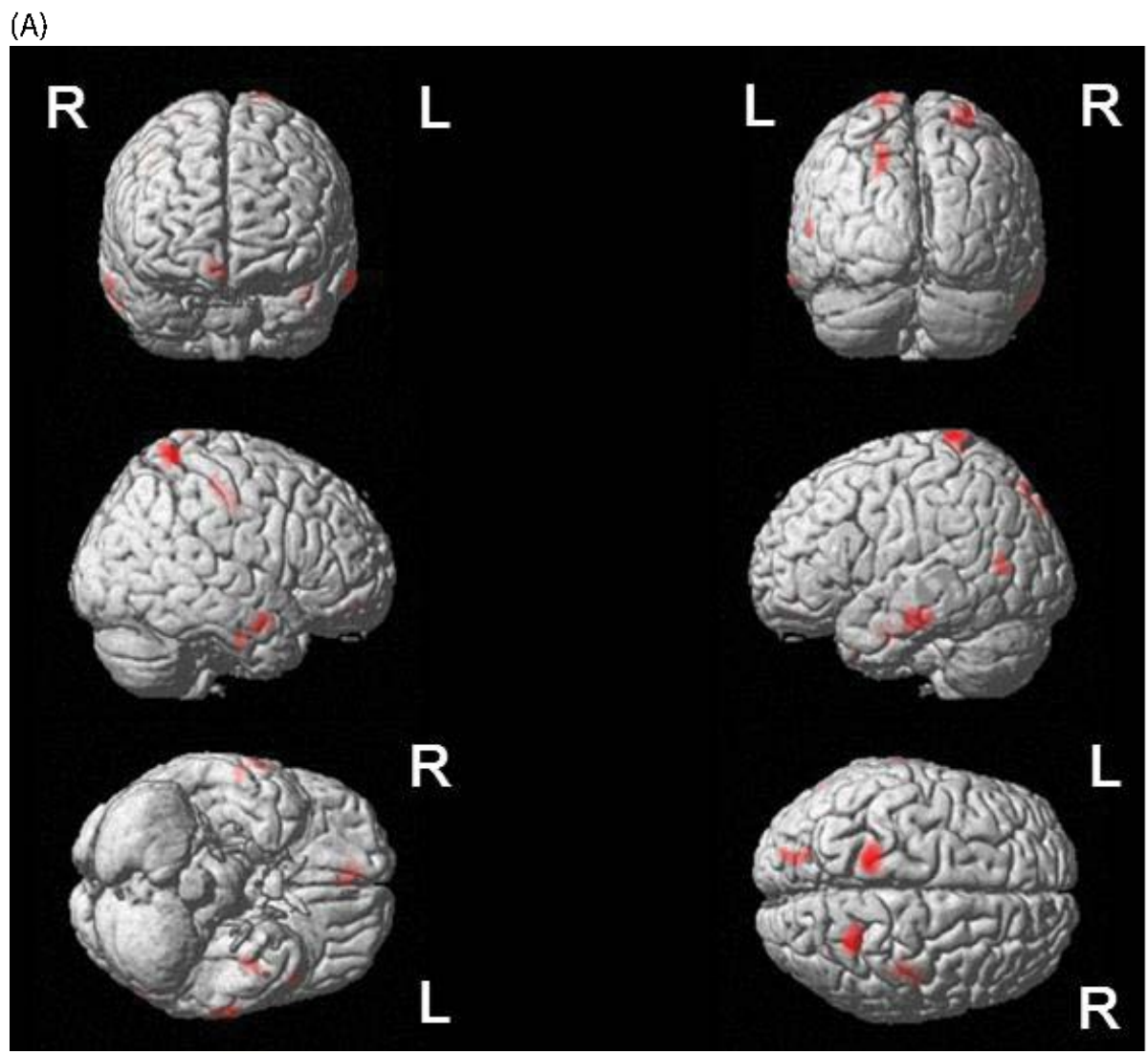

(B)
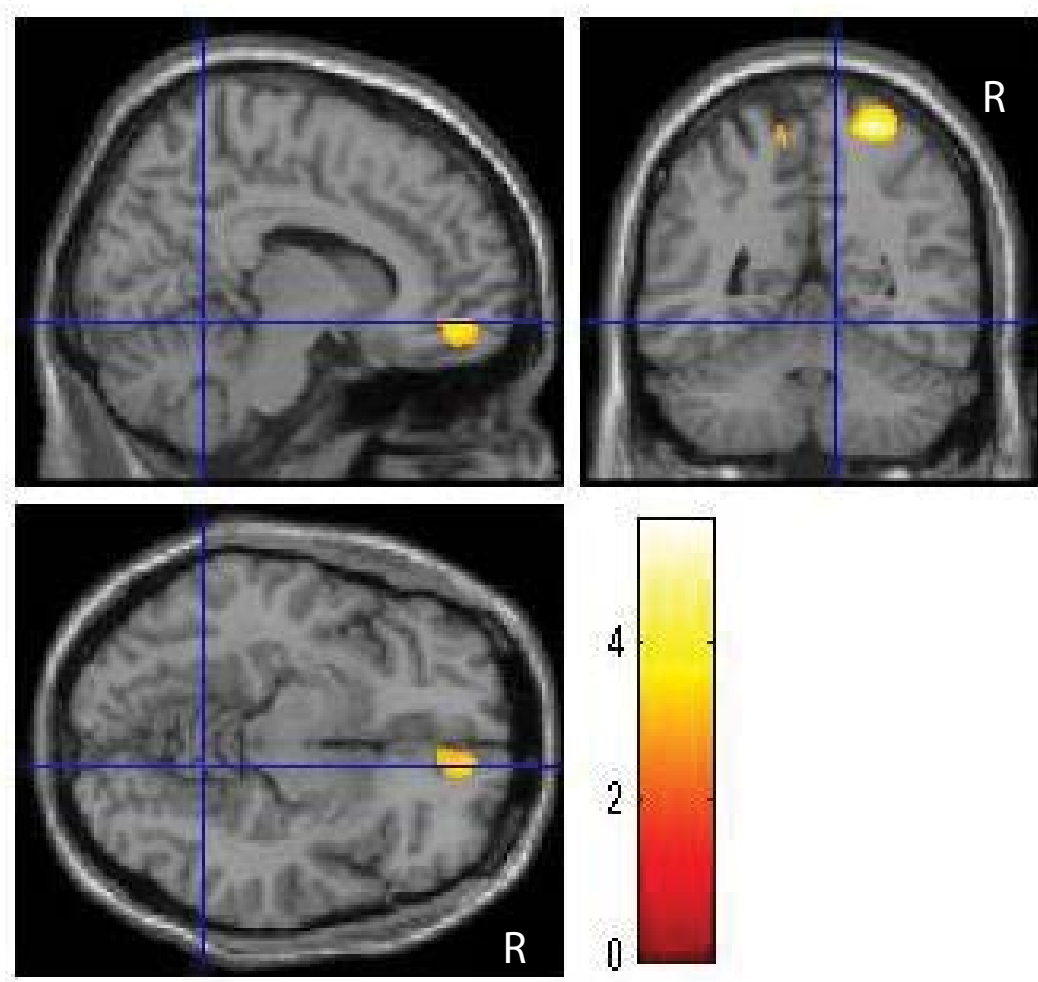

Figure 2: Statistical maps of higher cerebral blood flow after 12 weeks of memantine treatment compared with baseline.

Regions with significantly higher blood flow ( $p<0.01$; paired $t$-test) superimposed on a standard 3-dimensional anatomic template (3D-render) (A) and co-registered MRI slices (B). 
treatment alone. Additionally, we revealed that only a limited number of brain regions showed significant increases in cerebral blood flow after 12 weeks of sole memantine treatment in the same previously drug-naïve $\mathrm{AD}$ patients.

The regions of metabolic increases in this study were wider than those seen in the previous study by Sultzer et al. [21]. We speculate that the difference in the ranges of the two studies might be due to the different study designs. While we administered memantine to drug-naïve $\mathrm{AD}$ patients, Sultzer et al. added memantine treatment to patients on stable ChEI medication, and so the effect of memantine alone could not be assessed in their study. Actually, increased brain metabolism in left prefrontal cortex was demonstrated in a previous study of ChEI treatment [23]. Conversely, the metabolic increases in the angular and supramarginal gyri observed in our study were consistent with that observed by Sultzer et al. [21]. We speculate that the increased metabolic activity in these regions was due to memantine.

This is the first study to investigate the same drugnaïve $\mathrm{AD}$ patients before and after memantine treatment using 18F-FDG-PET and $99 \mathrm{mTc}$ ECD-SPECT. We found that the regions with increased activity on 18F-FDG-PET and 99mTc-ECD-SPECT were not consistent. This suggests that the increase in brain metabolism following memantine treatment is not directly caused by an increase in regional blood flow.

The targets of memantine, NMDARs, are one of the key players in pathophysiology of AD [24,25]. There are two types of NMDARs, synaptic NMDARs (sNMDARs) and extrasynaptic NMDARs (eNMDARs). It is believed that eNMDARs are linked to cell death signaling, and that $s$ NMDARs are associated with cell survival signal $[26,27]$. In $A D$ patients, $A \beta$ accumulation sequentially induces astrocytic glutamate release, increases of eNMDAR activity, and synaptic loss due to eNMDAR-mediated excitotoxicity [26,28-31]. Furthermore, eNMDAR activation impairs long-term potentiation through excessive $\mathrm{Ca}^{+}$influx, which also impairs neuronal plasticity $[32,33]$. The loss of synapses and the impairment of neuronal plasticity induced by eNMDAR activation are most likely the causes of learning and memory impairment [24,26].

The therapeutic mechanisms of memantine could involve preferentially blocking eNMDAR activation [34] and its downstream signaling, enhancing neuronal survival and synaptic plasticity, and suppressing the impairment of long term potentiation in brains of $\mathrm{AD}$ patients. We hypothesize that enhancement of neuronal survival and synaptic plasticity, and normalization of long term potentiation caused by blockage of eNMDAR, might increase brain metabolism before cerebral blood flow is increased by synaptic dysfunction recovery.

Chen et al. reported that the potentiation of brain-derived neurotrophic factor (BDNF) levels in serum and brain are observed in rats treated with low-dose memantine [35]. BDNF plays crucial roles in neuronal survival, neurotransmitter modulation, and leads to neuronal plasticity throughout its tyrosine kinase receptors $\mathrm{B}[36,37]$. In addition, $\mathrm{BDNF}$ shows neuroprotective activity by increasing sNMDAR activity and reducing eNMDAR activity [38]. Such neurotrophic effects of memantine might cause neuroprotection and neuro-regeneration, and inhibit memory impairment in $\mathrm{AD}$ patients.

Memantine has been reported to suppress the worsening of behavioral and psychological symptoms of Alzheimer's patients [1,2]. In our study, apparent trend in worsening of the patients at the end of the memantine treatment on the NPI score was observed (Table 1). The patients enrolled in our study did not show high behavioral and psychological symptoms at the base line (NPI score: 2.8), and one particular patient showed confusing increase of the NPI score. These may be one of the reasons for the apparent trend in worsening of NPI in our study.

There are limitations to our study. As this study was an open-label single arm exploratory study with a small number of patients $(\mathrm{n}=17)$, additional larger scale double-blind, placebocontrolled studies are necessary for further verification of our findings.

We believe this is the first study focusing on brain metabolic activity and perfusion in the same moderate $\mathrm{AD}$ patients before and after memantine treatment. Our findings suggest that memantine, independently from any increase in blood flow, improves brain metabolism in patients with moderate AD.

\section{Acknowledgements}

The authors thank all participants who took part in this study, and especially wish to thank Mr. Masahiro Oka and Mr. Kazuhito Kawasaki for 
technical assistance in conducting the PET and SPECT scans.

\section{Funding}

This study was funded by Daiichi-Sankyo Co., Ltd. The funders had no role in study design, data collection and analysis, decision to publish, or preparation of the manuscript.

\section{Competing and conflicting interests}

The authors declare that they have no competing interests.

\section{References}

1. Reisberg B, Doody R, Stoffler A, et al. Memantine in moderate-to-severe Alzheimer's disease. N. Engl. J. Med 348(14), 1333-1341 (2003).

2. Tariot PN, Farlow MR, Grossberg GT, et al. Memantine treatment in patients with moderate to severe Alzheimer's disease already receiving donepezil: a randomized trial. JAMA 201, 317-324 (2004).

3. Holman BL, Johnson KA, Gerada B, et al. The scientigraphic appearance of Alzheimer's disease: a prospective study using technetium-99m-HMPAO SPECT. J. Nucl. Med 33, 181-185 (1992).

4. Bartenstein P, Minoshima S, Hirsch C, et al. Quantitative assessment of cerebral blood flow in patients with Alzheimer's disease by SPECT. J. Nucl. Med 38(7), 1095-1101 (1997).

5. Bonte FJ, Weiner MF, Bigio EH, et al. SPECT imaging in dementias. J. Nucl. Med 42(7), 1131-1133 (2001).

6. Pagani M, Salmaso D, Ramstrom C, et al. Mapping pathological 99mTc-d, I-Hexamethylpropylene amine oxime uptake in $\mathrm{Alz}$ heimer's disease and frontal lobe dementia with SPECT. Dement. Geriatr. Cogn. Disord 12(1), 177-184 (2001).

7. Johnson KA, Holman BL, Mueller SP, et al. Single photon emission computed tomography in Alzheimer's disease. Abnormal iofetamine I 123 uptake reflects dementia severity. Arch. Neurol 45(4), 392-396 (1988).

8. Duara R, Grady C, Haxby J, Sundaram M, et al. Positron emission tomography in Alzheimer's disease. Neurology 36(7), 879887 (1986).

9. Herholz K, Carter SF, Jones M. Positron emission tomography imaging in dementia. Br. J. Radiol 80, S160-S167 (2007).

10. Engler $\mathrm{H}$, Forsberg A, Almkcst $\mathrm{O}$, et al. Two-year follow-up of amyloid deposition in patients with Alzheimer's disease. Brain 129, 2856-2866 (2006).

11. Hirono N, Hashimoto $M$, Ishii $K$, et al. Oneyear change in cerebral glucose metabolism in patients with Alzheimer's disease. Am. J. Psychiatry Clin. Neurosci 16, 488-492 (2004).

12. Alexander G, Chen $K$, Pietrini $P$, et al. Longitudinal PET Evaluation of cerebral metabolic decline in Dementia: A potential Outcome measure in Alzheimer's disease treatment studies. Am. J. Psychiatry 159, 738-745 (2002).

13. Stefanova E, Wall A, Almkvist O, et al. Longitudinal PET evaluation of cerebral glucose metabolism in rivastigmine treated patients with mild Alzheimer's disease. J. Neural. Transm 113(2), 205-218 (2006).

14. Ito $K$, Shimano $Y$, Imabayashi $E$, et al. Concordance between (99m)Tc-ECD SPECT and 18F-FDG PET interpretations in patients with cognitive disorders diagnosed according to NIA-AA criteria. Int. J. Geriatric Psychiatry 29(10), 1079-1086 (2014).

15. Shimizu S, Kaneta $H$, Hirose D, et al. Differential effects of acetylcholinesterase inhibitors on clinical responses and cerebral blood flow changes in patients with Alzheimer's disease: A 12-month, randomized, and open-label trial. Dement. Geriatr. Cogn. Disord. Extra 5, 135-146 (2015).

16. Cerci S, Tamam Y, Kaya H, et al. Effect of Rivastigmine on regional cerebral blood flow in Alzheimer's disease. Advances in Therapy 24(3), 611-621 (2007).

17. Ceravolo R, Volterrani D, Tognoni G, et al. Cerebral perfusional effects of cholinesterase inhibitors in Alzheimer's disease. Clin. Neuropharmacol 27(4), 166170 (2004).

18. Nakano S, Asada T, Matsuda H, et al. Donepezil hydrochloride preserves regional cerebral blood flow in patients with Alzheimer's disease. J. Nucl. Med 42(10), 1441-1445 (2001)

19. Nobili F, Koulibaly M, Vitali P, et al. Brain perfusion follow-up in Alzheimer's patients during treatment with acetylcholinesterase inhibitors. J. Nucl. Med 43(8), 983-990 (2002).

20. Keller C, Kadir A, Forsberg A, et al. Longterm effects of galantamine treatment on brain functional activities as measured by PET in Alzheimer's disease patients. J. Alzheimer's Disease 24(1), 109-123 (2011).

21. Sultzer D, Melrose R, Harwood D, et al. Effect of memantine treatment on regional cortical metabolism in Alzheimer's disease. Am. J. Geriatr. Psychiatry 18(1), 606-614 (2010).

22. Cummings JL, Mega M, Gray K, et al. The
Neuropsychiatric Inventory: comprehensive assessment of psychopathology in dementia. Neurology 44(12), 2308-2314 (1994).

23. Teipel SJ, Drzezga A, Bartenstein P, et al. Effects of donepezil on cortical metabolic response to activation during (18) FDG-PET in Alzheimer's disease: a double-blind cross-over trial. Psychopharmacology 187(1), 86-94 (2006).

24. Zhang Y, Li P, Feng J, et al. Dysfunction of NMDA receptors in Alzheimer's disease. Neurol. Sci 37(7), 1039-47 (2016).

25. Banding $\mathrm{H}$. Therapeutic targeting of the pathological triad of extrasynaptic NMDA receptor signaling in neurodegenerations. J. Exp. Med 214(3) 569-578 (2017).

26. Parsons MP, Raymond LA. Extrasynaptic NMDA receptor involvement in central nervous system disorders. Neuron 82(1), 279-293 (2014).

27. Huang Y, Shen W, Su J, et al. Modulating the balance of synaptic and extrasynaptic NDMA receptors shows positive effects against amyloid- $\beta$-induced neurotoxicity. J. Alzheimer's Dis 57(3), 885-897 (2017).

28. Hardy J, Selkoe DJ. The amyloid hypothesis of Alzheimer's disease: progress and problems on the road to therapeutics. Science 297(5580), 353-356 (2002).

29. Parameshwaran K, Dhanasekaran M, Suppiramaniam V. Amyloid beta peptides and glutamatergic synaptic dysregulation. Exp. Neurol 210(1), 7-13 (2008).

30. Jack CR Jr, Knopman DS, Jagust WJ, et al. Hypothetical model of dynamic biomarkers of the Alzheimer's pathological cascade. Lancet Neurol 9(1), 119 (2010).

31. Talantova $M$, Sanz-Blasco $S$, Zhang $X$, et al. $A \beta$ induces astrocytic glutamate release, extrasynaptic NMDA receptor activation, and synaptic loss. Proc. Natl. Acad. Sci. US A 110(27), E2518-2527 (2013).

32. Hanson JE, Pare JF, Deng $L$, et al. Altered GluN2B NMDA receptor function and synaptic plasticity during early pathology in the PS2APP mouse model of Alzheimer's disease. Neurobiol. Dis 74, 254-262 (2015).

33. Li S, Jin $M$, Koeglsperger T, et al. Soluble $A \beta$ oligomers inhibit long-term potentiation through a mechanism involving excessive activation of extrasynaptic NR2B-con- 
Effect of Memantine on Brain Metabolic Activity and Perfusion in Drug-naïve Moderate Alzheimer's Disease Patients

taining NMDA receptors. J. Neurosci 31(18) 6627-6638 (2011).

34. Xia P, Chen HS, Zhang D, et al. Memantine preferentially blocks extrasynaptic over synaptic NMDA receptor currents in hippocampal autapses. J. Neurosci 30(33), 11246-11250 (2010).

35. Chen SL, Tao PL, Chu CH, et al. Low-dose memantine attenuated morphine addictive behavior through its anti-inflammation and neurotrophic effects in rats. J. Neuroimmune Pharmacol 7, 444-453 (2001).

36. Harward SC, Hedrick NG, Hall CE, et al. Autocrine BDNF-TrkB signalling within a single dendritic spine. Nature 538(7623), 99-103 (2016).

37. Kocahan S, Doğan Z. Mechanisms of Alzheimer's disease pathogenesis and prevention:

\section{Research}

the brain, neural pathology, N-methyl-D-aspartate receptors, tau protein and other risk factors. Clin. Psychopharmacol. Neurosci 15(1), 1-8 (2017)

38. Lau D, Benqtson CP, Buchthal B, et al. BDNF reduces toxic extrasynaptic NMDA receptor signaling via synaptic NMDA receptors and nuclear-calcium-induced transcription of inhba/activin A. Cell Rep 12(8) 1353-1366 (2015). 